\title{
Molecular characterization of MHC-DRB cDNA in water buffalo (Bubalus bubalis)
}

\author{
Soumen Naskar ${ }^{1}$, Sitangsu M. Deb ${ }^{2}$, Saket K. Niranjan ${ }^{3}$, Subodh Kumar, Deepak Sharma ${ }^{4}$, \\ Durgam Sakaram $^{5}$ and Arjava Sharma ${ }^{6}$ \\ Animal Genetics Division, Indian Veterinary Research Institute, Izatnagar, Uttar Pradesh, India.
}

\begin{abstract}
In the present study, water buffalo MHC (Bubu)-DRB cDNA was cloned and characterized. The 1022 base long-amplified cDNA product encompassed a single open reading frame of 801 bases that coded for 266 amino acids. The Bubu-DRB sequence showed maximum homology with the BoLA-DRB3*0101 allele of cattle. A total of seven amino acid residues were found to be unique for the Bubu-DRB sequence. The majority of amino acid substitutions was observed in the $\beta_{1}$ domain. Residues associated with important functions were mostly conserved. Water buffalo $D R B$ was phylogenetically closer to goat $D R B^{\star} A$.
\end{abstract}

Key words: water buffalo, cDNA, DRB, MHC, phylogeny.

Received: July 13, 2011; Accepted: October 26, 2011.

The major histocompatibility complex (MHC) plays a central role in the processing and presentation of antigens, and in discriminating between self and non-self. MHC class II molecules, after selectively binding different peptides derived from exogenous pathogens, present them to helper T cells (Brown et al., 1993). MHC class II molecules, located primarily on the surface of antigen-presenting cells, are heterodimer glycoproteins composed of two polymorphic transmembrane polypeptide chains, termed $\alpha$ and $\beta$. The $D R B$ genes under the DR region of the MHC complex encode for the highly polymorphic $\beta$-chain (Ellis and Ballingall, 1999). Three bovine $D R B$ genes have been reported. Of these, $D R B 3$ appears to be highly expressed, $D R B 1$ is a pseudogene, and $D R B 2$ is transcribed at a very low level in lymphocyte tissues (Lewin et al., 1999). An extremely polymorphic second exon of the $D R B$ gene encodes those peptide binding sites (PBS) responsible for antigen binding (Rask et al., 1985). Currently, more than 100 alleles of the $D R B 3$ gene have been described (BoLA

Send correspondence to Soumen Naskar. National Research Centre on Pig, Rani, Guwahati 781131 Assam, India. E-mail: snrana@gmail.com.

*Present addresses:

${ }^{1}$ National Research Centre on Pig, Guwahati, Assam, India.

${ }^{2}$ Central Arid Zone Research Institute Regional Research Station, Pali-Marwar, Rajasthan, India.

${ }^{3}$ National Bureau of Animal Genetic Resources, Karnal, Haryana, India.

${ }^{4} \mathrm{Pt}$. Deen Dayal Upadhayaya Veterinary and Animal Sciences University, Mathura, Uttar Pradesh, India.

${ }^{5}$ Sri Venkateswara Veterinary University, Hyderabad, Andhra Pradesh, India.

${ }^{6}$ Project Directorate on Cattle, Meerut, Uttar Pradesh, India.
Nomenclature, ISAG; EMBL-EBI) in Bos taurus and Bos indicus cattle. Importantly, besides its association with several diseases, polymorphism at the $D R B$ exon 2 region is also considered a potential candidate-marker gene.

Riverine buffalo (Bubalus bubalis), well adapted to a hot and humid tropical climate, seems to tolerate many common infectious diseases. Although already mapped on chromosome 2 (Rodrigues et al., 2008), water-buffalo MHC has been little studied, with the scarcity of data regarding diversity (De et al., 2002, Sena et al., 2003, Niranjan et al., 2009). Herein, DRB genes expressed in water buffalo were cloned and characterized.

Venous blood was collected from a randomly selected and healthy Indian Murrah water buffalo. Total RNA was extracted from a lymphocyte culture with total RNA minipreps super kit (Biogene, USA). RT-PCR was carried out with a RevertAid first strand cDNA kit (MBI Fermentas). Both strands of $D R B$ cDNA were produced by PCR, using Forward - 5'-GACTTGCCTGCTCCTCTCAC TCTC-3' and Reverse - 5'-AGGGTGGGGGTGACTGAG AAAAC-3' primers, with design based on the cattle DRB 3 sequence (Accession No. AY125893). PCR amplification was in a total volume of $25 \mu \mathrm{L}$ with $6 \mu \mathrm{L}$ of first strand cDNA, 1x PCR buffer, $1.5 \mathrm{mM}$ of $\mathrm{MgCl}_{2}, 200 \mu \mathrm{M}$ of each dNTPs, 30 pmol of each primer and 1 unit of Taq DNA polymerase. The polymerase chain reaction (PCR) was carried out in a thermal cycler (DNA Engine PTC200, M.J. Research) under the following conditions: initial denaturation at $94{ }^{\circ} \mathrm{C}$ for $3 \mathrm{~min}$, followed by 35 cycles of $94{ }^{\circ} \mathrm{C}$ for $1 \mathrm{~min}, 60^{\circ} \mathrm{C}$ for $1 \mathrm{~min}$ and $72^{\circ} \mathrm{C}$ for $1.5 \mathrm{~min}$, and a final extension at $72{ }^{\circ} \mathrm{C}$ for $10 \mathrm{~min}$. The amplified cDNA product 
of $D R B$ was purified using the QIAquick gel extraction kit (Qiagen). The purified product was ligated into a pGEM-T Easy vector system (Promega). After confirmation by colony PCR, and the release of inserts from plasmids by digestion with $E c o R$ I, positive clones were subjected to double stranded sequencing by primer walking in an automated DNA sequencer. The obtained sequence was annotated and submitted to NCBI GenBank (Acc. no. DQ057985). The nucleotide and deduced amino acid sequences of water buffalo DRB cDNA were compared with those from other species.

Based on the $D R B$ cDNA sequences of the water buffalo and other species, the neighbor-joining method together with the MEGA 4 programme (Tamura et al., 2007) was applied for defining a phylogenetic tree. Bootstrap values were obtained using 500 replicates. Evolutionary divergence (base substitution/site) was computed by the bootstrap procedure (500 replicates) using the Kimura 2-parameter method based on a nucleotide model (Kimura, 1980) from the MEGA 4 programme (Tamura et al., 2007).

The amplified 1022-nucleotide-long cDNA sequence of the water buffalo $D R B$ gene contained the complete cod- ing region of 801 nucleotides. The annotated Bubu- $D R B$ sequence was highly similar to orthologous $D R B$ sequences of other species. Homology with various cattle alleles varied between $89.9 \%(B o L A-D R B 3 * 3101)$ and $92.1 \%$ (BoLA-DRB3*0101). Homologies with sheep, goat, pig and human alleles were 89.8, 89.1, 77.5 and $79 \%$, respectively.

The resultant Bubu-DRB amino acid sequence was 266 amino acids long. Six exons encoding six different domains, a characteristic feature of MHC class II molecules, were confirmed (Russel et al., 1997). The sizes of various domains, viz., signal peptide, $\beta_{1}, \beta_{2}$, connecting peptide (CP), transmembrane (TM) and cytoplasmic (CY) domains, were analogous to the $D R B$ sequences of other species (Figure 1). A total of seven amino acid residues $(3,29$, $66,79,150,153$ and 227) were unique to the $B u b u-D R B$ sequence, six of which were otherwise highly conserved. Importantly, one of the residues (position 66) was associated with an antigen binding site (ABS). When compared with the closest cattle allele, $D R B 3^{*} 0101,20$ residue substitutions with maximum numbers (11) in the $\beta_{1}$ domain of $B u b u-D R B$ were observed. The water buffalo sequence

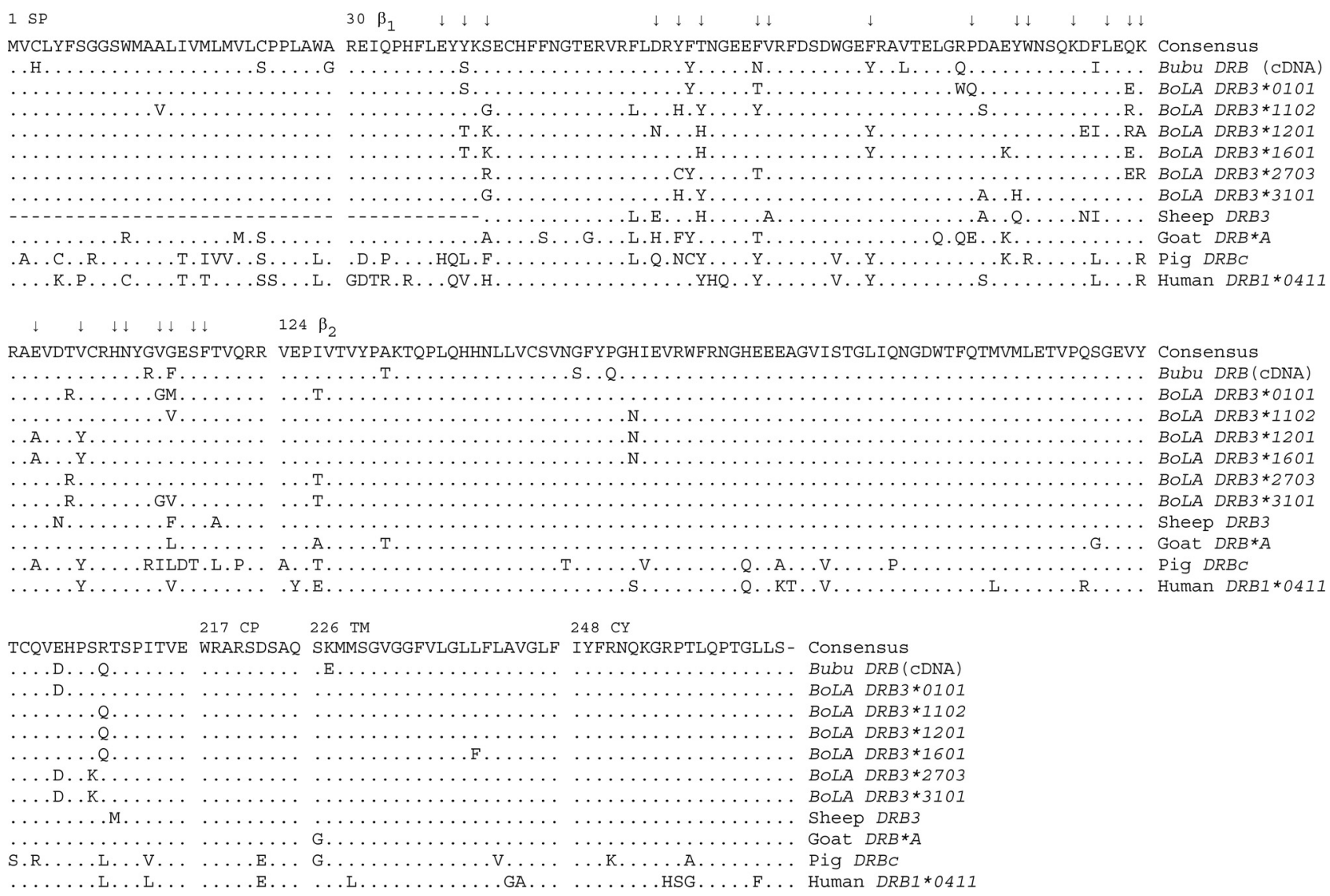

Figure 1 - Comparison between the predicted amino acid sequences of water buffalo $D R B$ cDNA, and the corresponding sequences from other species. Identity is denoted by dots (.) and non-identical amino acids are indicated. Gaps, represented by a dash (-), were introduced to optimize similarity. A ‘ $\downarrow$ ' sign indicates an antigen binding site. Species and accession numbers are: water buffalo (Bubu-DRB; DQ057985), cattle [BoLA-DRB3*0101 (X92409), *1102 (Y18308), *1201 (AY125893), *1601 (D45357), *2703 (AY125892) and *3101 (U77067)], sheep DRB3 (L04790), goats DRB*A (AB008345), pigs $D R B C(\mathrm{M} 55165)$ and humans $D R B 1 * 0411$ (AY961063). 
showed 22, 26, 57 and 51 residue-differences with those of sheep, goats, pigs and humans, respectively. As expected, most of the substitutions were observed in the $\beta_{1}$ domain when compared across species. Alleles at the BoLA-DRB3 locus gave evidence of over-dominant selection, with the peptide binding site (PBS) region presenting a higher proportion of mutations compared to non-PBS regions (Sena et al., 2003).

169 residues (SP-13, $\beta_{1}-49, \beta_{2}-70$, CP- 8 , TM- 15 and CY-14) were noted to be completely conserved across the species. Among these, 49 were found to be specific to ruminants, but not to pig and human. Out of the 24 residues associated with ABS (Brown et al., 1993), in water buffalo, 17 were found to be conserved, and those at positions 40, 42, 61, 66, 100, 107 and 115 to be different. Valine (115), essential in creating a deep pocket at PBS surrounded by predominantly nonpolar residues of both the $\alpha_{1}$ and $\beta_{1}$ domains, was found substituted by phenylalanine in water buffalo. Interspecies variability of this residue is great. The dominant 'anchoring' peptide residue binds to the hydrophobic pocket of Phe (115), along with $\alpha_{26}$ and $\alpha_{31}$. One of the residues (84) associated with CD4 binding (Brown et $a l ., 1988)$, was found to be substituted in water buffalo $D R B$. Highly conserved Asn (111), which supposedly forms the hydrogen bond through its $\gamma$ amide group with the main chain peptide, was invariant in water buffalo. Mutation at this position affects intracellular transport and cellsurface expression of class II molecules in mice. Similarly, the residue Trp (90), apparently conserved across species, is placed in such a way that its indole nitrogen is capable of donating a hydrogen bond to the main chain carbonyl group of a bound peptide, in exact homology to the location and function of Trp (147) of class I. Polymorphic residue Asp (86) forms a salt bridge with a conserved Arg (76) of the $\alpha$ chain under the existing peptide (Nydam et al., 1998). This residue $\left(\beta_{57}\right)$ of $H L A-D Q$ has been found associated with insulin-dependent diabetes mellitus (IDDM) in humans (Brown et al., 1993). In addition to any possible effect on peptide binding, polymorphism at this residue may affect either the stability or structure of the $\alpha \beta$ heterodimer, since nearby residues Glu (81) and Arg (84) form part of the interface of the dimer (Brown et al., 1988).

Two salt bridges from Glu (81) to Arg (84) on the neighboring molecule are conserved in $H L A-D R 1$, which forms the first of four interfaces between DR1 molecules in dimers. However, residue Arg (84) was found to be substituted by Gln in water buffalo. Three more salt bridges, i.e., His (140), Glu (191) and His (141) of the $\beta_{2}$ domain, with the $\alpha_{2}$ domain residues Glu (158), His (177) and Glu (179), respectively, were found to be conserved across the species. The T-cell coreceptor molecule, CD4, is thought to bind to the residues, Gln (139), Glu (166), Ala (169), Gly (170) and Val (171). With the exception of residue Ala (169) in humans (Ala $\rightarrow \mathrm{Thr}$ ), total conservation across species was observed in these positions. Apart from a partial affinity for Gln (139), residues Gly (170) and Val (171) are very important for CD4 binding.

In the mechanism by which MHC molecules tightly bind several peptides, the role of clusters of conserved residues in the class II molecules, Trp (91) (except Arg in pig) and Asn (111) (along with $\alpha_{62}$ Asn, $\alpha_{69}$ Asn and $\alpha_{76}$ Arg), are spaced so as to bind at intervals to the extended main chain of bound peptides, thereby providing an independent peptide side chain component to the affinity (Brown et al., 1988). The disulphide linked Cys residues at positions 44 and 108 of the $\beta_{1}$ domain, and at positions 146 and 202 of the $\beta_{2}$, were conserved across species. The N-glycosylation region, or the - $\mathrm{CHO}$ site at residue positions 48-50 (AsnGly-Thr), was completely conserved in all the species compared.

Phylogenetic analysis based on complete cDNA using the Neighbor-Joining method (Nucleotide: Kimura 2-parameter model), revealed the gathering of several ruminant $D R B$ alleles into a single group (Figure 2). The clustering of different ruminant sequences along with those of water buffalo seems to have arisen from trans-species evolution, a feature common to MHC genes, where the clusters had possibly ensued from primordial sequences in a common ancestor (Klein, 1986). Similarly, the sharing of common types of pathogens among the ruminants is also indicated (Hickford et al., 2004). Nevertheless, Bubu-DRB seems to be closer to the goat $D R B$ sequence than to those of cattle. Evolutionary divergence of $B u b u-D R B$ based on base substitution per site was the lowest $(0.036)$ with $B o L A-D R B 3^{*} 0101(0.036)$ and the highest (0.139) in pig (Table 1).

Thus, it is apparent that $B u b u-D R B$ is expressed, and that there is considerable similarity with other ruminant

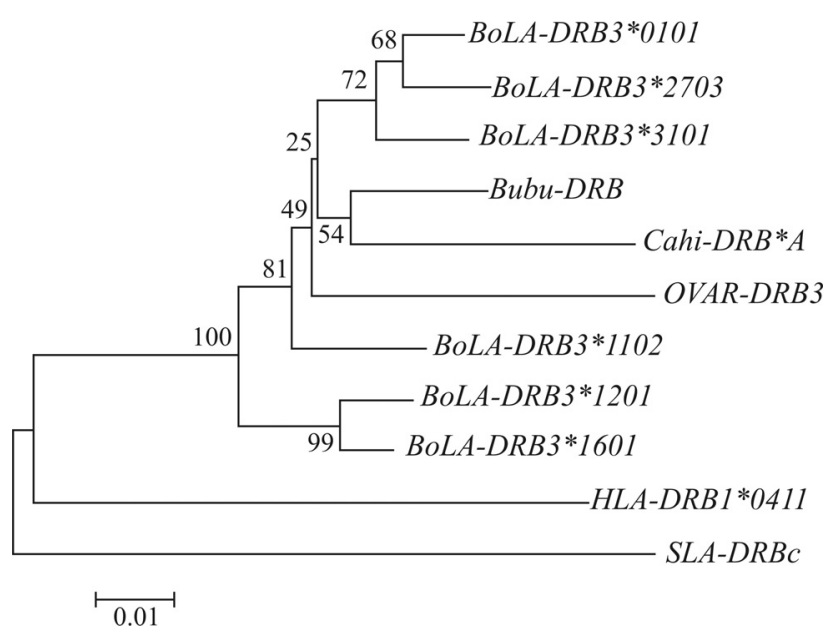

Figure 2 - Phylogenetic tree, compiled by using the Neighbor-Joining method (p-distance model: Kimura 2-parameter method) based on cDNA sequences of $D R B$ genes from different species (as described in Figure 1). Branch lengths are in the same units as those used for evolutionary distances. The number at each branch-point indicates the bootstrap (500) confidence level. 
Table 1 - Evolutionary divergence of the buffalo $(B u b u)$ - $D R B$ cDNA sequence from those of other species (base substitution/site) computed by the bootstrap procedure (500 replicates), using the Kimura 2-parameter method, based on the nucleotide model in the MEGA 4 programme (Tamura et al., 2007). Accession No(s). for the $D R B$ sequences from different species are described in Figure 1.

\begin{tabular}{lc}
\hline Sequence & Base substitution/site \\
\hline$B o L A-D R B 3 * 0101$ & $0.036 \pm 0.007$ \\
$B o L A-D R B 3 * 1102$ & $0.044 \pm 0.008$ \\
$B o L A-D R B 3 * 1201$ & $0.050 \pm 0.008$ \\
$B o L A-D R B 3 * 1601$ & $0.052 \pm 0.008$ \\
$B o L A-D R B 3 * 2703$ & $0.039 \pm 0.007$ \\
$B o L A-D R B 3 * 3101$ & $0.047 \pm 0.008$ \\
OVAR-DRB3 & $0.065 \pm 0.009$ \\
Cahi-DRB*A & $0.053 \pm 0.009$ \\
SLA-DRBC & $0.139 \pm 0.014$ \\
HLA-DRB $* 0411$ & $0.128 \pm 0.014$ \\
\hline
\end{tabular}

species, not only as to cDNA sequences, but also functionally important conserved residues. Nevertheless, high genetic variation corresponding to the exon 2 region of cDNA, possibly supplementary to antigen repertoire properties, was revealed.

\section{Acknowledgments}

The authors wish to thank the Director of the Indian Veterinary Research Institute, Izatnagar, for the use of facilities. Financial assistance provided by the Department of Biotechnology (Government of India) is duly acknowledged.

\section{References}

Brown JH, Jardetzky TS, Saper MA, Samraoui B, Bjorkman PJ and Wiley DC (1988) A hypothetical model of the foreign antigen binding site of class II histocompatibility molecules. Nature 332:845-850.

Brown JH, Jardetzky TS, Gorga JC, Stern LJ, Urban RG, Strominger JL and Wiley DC (1993) Three-dimensional structure of the human class II histocompatibility antigen HLA-DR1. Nature 364:33-39.

De S, Singh RK and Butchaiah G (2002) MHC-DRB exon 2 allele polymorphism in Indian river buffalo (Bubalus bubalis). Anim Genet 33:215-219.

Ellis SA and Ballingall KT (1999) Cattle MHC: Evolution in action. Immunol Rev 167:159-168.
Hickford JGH, Zhou H, Slow S and Fang Q (2004) Diversity of ovine DQA2 gene. Anim Genet 82:1553-1563.

Kimura M (1980) A simple method for estimating evolutionary rate of base substitutions through comparative studies of nucleotide sequences. J Mol Evol 16:111-120.

Klein J (1986) Natural History of the Major Histocompatibility Complex. John Wiley and Sons, New York, 775 pp.

Lewin HA, Russell GC and Glass EJ (1999) Comparative organization and function of the MHC of domestic cattle. Immunol Rev 167:145-158.

Niranjan SK, Deb SM, Sharma A, Mitra A and Kumar S (2009) Isolation of two cDNAs encoding MHC-DQA1 and DQA2 from the water buffalo, Bubalus bubalis. Vet Immunol Immunopathol 130:268-271.

Nydam T, Wade TK, Yadati S, Gabriel JL, Barisas BG and Wade WF (1998) Mutations in MHC class II dimer of dimers contact residues: Effect on antigen presentation. Int Immunol 10:1237-1249.

Rask I, Gusafsson K, Larhammer D, Ronne H and Peterson PA (1985) Generation of class II antigen polymorphism. Immunol Rev 84:123-143.

Rodrigues EAF, Stafuzza NB, Caetano AR, Gill CA, Riggs PK, Womack JE and Amaral ME (2008) Mapping MHC genes in river buffalo. Dev Biol 132:343-346.

Russel GC, Davies CJ, Andersson L, Ellis SA, Hensen EJ, Lewin HA, Mikko S, Muggli-Cockett NE and Van der Poel JJ (1997) BoLA class II nucleotide sequences, 1996: Report of the ISAG BoLA Nomenculature Committee. Anim Genet 28:169-180.

Sena L, Schneider MPC, Brenig B, Honeycutt RL, Womach JE and Skow LC (2003) Polymorphisms in MHC-DRA and DRB alleles of water buffalo (Bubalus bubalis) reveal different features from cattle DR alleles. Anim Genet 34:1-10.

Tamura K, Dudley J, Nei M and Kumar S (2007) MEGA4: Molecular Evolutionary Genetics Analysis (MEGA) software version 4.0. Mol Biol Evol 24:1596-1599.

\section{Internet Resources}

Bovine Leukocyte Antigen (BoLA) Nomenclature, International Society for Animal Genetics (ISAG).

http://www.projects.roslin.ac.uk/bola/bolahome.html (June 25, 2011).

European Molecular Biology Laboratory - European Bioinformatics Institute (EMBL-EBI).

http://www.ebi.ac.uk/cgi-bin/ipd/mhc/ (June 25, 2011).

Associate Editor: Bertram Brenig

License information: This is an open-access article distributed under the terms of the Creative Commons Attribution License, which permits unrestricted use, distribution, and reproduction in any medium, provided the original work is properly cited. 